\title{
Entrapment of curcumin into monoolein-based liquid crystalline nanoparticle dispersion for enhancement of stability and anticancer activity
}

This article was published in the following Dove Press journal:

International Journal of Nanomedicine

26 June 2014

Number of times this article has been viewed

\section{Rengarajan Baskaran' \\ Thiagarajan Madheswaran ${ }^{2}$ \\ Pasupathi Sundaramoorthy' \\ Hwan Mook Kim' \\ Bong Kyu Yoo' \\ 'College of Pharmacy, Gachon University, Incheon, South Korea; ${ }^{2}$ College of Pharmacy Yeungnam University, Gyeongsan, South Korea}

Correspondence: Bong Kyu Yoo

College of Pharmacy, Gachon University,

191 Hambakmoero, Incheon 406-799,

South Korea

Tel +82 328996415

Fax +82328204829

Email byoo@gachon.ac.kr

\begin{abstract}
Despite the promising anticancer potential of curcumin, its therapeutic application has been limited, owing to its poor solubility, bioavailability, and chemical fragility. Therefore, various formulation approaches have been attempted to address these problems. In this study, we entrapped curcumin into monoolein (MO)-based liquid crystalline nanoparticles (LCNs) and evaluated the physicochemical properties and anticancer activity of the LCN dispersion. The results revealed that particles in the curcumin-loaded LCN dispersion were discrete and monodispersed, and that the entrapment efficiency was almost $100 \%$. The stability of curcumin in the dispersion was surprisingly enhanced (about $75 \%$ of the curcumin survived after 45 days of storage at $\left.40^{\circ} \mathrm{C}\right)$, and the in vitro release of curcumin was sustained $(10 \%$ or less over 15 days). Fluorescence-activated cell sorting (FACS) analysis using a human colon cancer cell line (HCT116) exhibited $99.1 \%$ fluorescence gating for $5 \mu \mathrm{M}$ curcumin-loaded LCN dispersion compared to $1.36 \%$ for the same concentration of the drug in dimethyl sulfoxide (DMSO), indicating markedly enhanced cellular uptake. Consistent with the enhanced cellular uptake of curcumin-loaded LCNs, anticancer activity and cell cycle studies demonstrated apoptosis induction when the cells were treated with the LCN dispersion; however, there was neither noticeable cell death nor significant changes in the cell cycle for the same concentration of the drug in DMSO. In conclusion, entrapping curcumin into MO-based LCNs may provide, in the future, a strategy for overcoming the hurdles associated with both the stability and cellular uptake issues of the drug in the treatment of various cancers.
\end{abstract}

Keywords: liquid crystalline nanoparticle, anticancer activity, curcumin, monoolein, cellular uptake, cell cycle distribution

\section{Introduction}

Cancer remains one of the most devastating diseases to modern society, with an annual incidence of more than 10 million cases. ${ }^{1}$ Current anticancer treatments consist of surgical interventions, irradiation, and chemotherapy, with the last two treatment modalities causing significant adverse events, owing to toxicity toward normal cells and tissues. Therefore, developing a chemotherapeutic agent that selectively kills malignant cells while leaving normal cells unharmed would help reduce adverse effects.

Curcumin, a well-known dietary pigment from the turmeric plant (Figure 1), has been found to inhibit the in vivo and in vitro growth of several malignant cell types, showing no harm to normal cells. ${ }^{2-5}$ Specifically, curcumin-induced apoptosis in human breast tumor cell lines (MCF-7 and MDAMB), and in a human hepatocellular carcinoma cell line (HepG2), in a dose-dependent and time-dependent manner. ${ }^{6}$ Curcumin has also been shown to possess apoptotic activity against human colon cancer cells, ${ }^{7}$ stomach and skin tumors, ${ }^{8}$ and prostate cancer cells. ${ }^{9}$ 


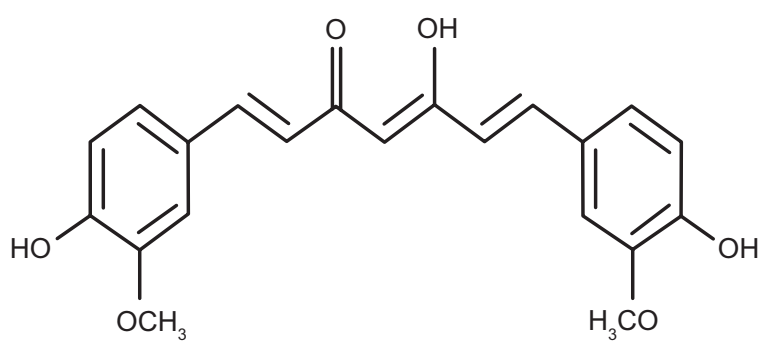

Figure I Chemical structure of curcumin.

However, despite the promising anticancer potential of curcumin, its therapeutic application has been limited because of its poor solubility, bioavailability, and chemical fragility. ${ }^{10,11}$ In order to address these drawbacks, formulation approaches using solid dispersion, ${ }^{12}$ liposomes, ${ }^{13}$ cyclodextrin complexes, ${ }^{14,15}$ and nanoparticles have been reported. ${ }^{16-18}$ Although some progress has been achieved, these approaches have issues of poor drug loading, drug leakage, burst release patterns, and poor physicochemical stability that are unaddressed.

In this context, liquid crystalline nanoparticles (LCNs) have attracted significant attention due to their potential improvements in physicochemical stability, hydrophobic drug loading, sustained release patterns, and a reduction in drug leakage. Earlier, we reported that hydrophobic drugs were efficiently entrapped into monoolein (MO)-based LCNs and that the release of the drugs was sustained up to several weeks without a burst effect. ${ }^{19,20}$ Furthermore, we were able to control the release of the drug by incorporating hydrophilic and hydrophobic additives into the LCN. ${ }^{21}$

In this study, we prepared curcumin-loaded LCNs (LCN-R [liquid crystalline nanoparticles prepared with Cremophor RH40 as a solubilizer]) with the aid of drug solubilizers and investigated the effect of the LCN on cellular uptake, anticancer activity, and cell cycle distribution on a human colonic cancer cell line (HCT116). Characterization of the LCN, drug release, and the physicochemical stability of the LCN were also performed.

\section{Materials and methods \\ Materials}

MO was received as a gift from Danisco Ltd. (Tokyo, Japan). Poloxamer 407 (P 407) and Cremophor ${ }^{\circledR}$ RH 40 (RH 40) were purchased from BASF SE (Ludwigshafen, Germany). Curcumin and polyethyleneglycol 400 (PEG 400) were purchased from Sigma-Aldrich Co. (St Louis, MA, USA). The human colon cancer cell line (HCT116) was purchased from the American Type Culture Collection (Manassas, VA, USA). Penicillin, streptomycin, and Roswell Park Memorial
Institute (RPMI)-1640 medium were purchased from Gibco $^{\circledR}$ (Thermo Fisher Scientific, Waltham, MA, USA). A Spectra/ Por $^{\circledR}$ dialysis membrane was purchased from Spectrum Laboratories Inc. (Rancho Dominguez, CA, USA). All other chemicals were of reagent grade and were used without further purification.

\section{Solubility of curcumin}

The solubility of curcumin in various vehicles was determined by the shake-flask method. An excess amount of curcumin was added into a vial containing $1 \mathrm{~mL}$ of the vehicle and was vortexed for 5 minutes. The mixture was shaken in a water bath, while maintaining the temperature at $37^{\circ} \mathrm{C}$ for 24 hours, and then centrifuged at 12,000 rpm for 5 minutes, followed by filtration through a syringe filter to remove the undissolved curcumin particles. The filtrate was diluted with methanol and the amount of solubilized curcumin was measured using high-performance liquid chromatography (HPLC), as described in the following text.

\section{Preparation of curcumin-loaded LCN dispersion}

LCN dispersion was prepared as described previously, with slight modifications. ${ }^{22}$ Briefly, $30 \mathrm{mg}$ of curcumin was solubilized into PEG 400, RH 40, or a combination of the two vehicles. The curcumin solution was slowly added to a small vial containing melted $\mathrm{MO}(1.2 \mathrm{~g})$, while maintaining the temperature at $45^{\circ} \mathrm{C}$ (solubilizer concentration: $50 \%$ with respect to $\mathrm{MO})$. The mixture of $\mathrm{MO}$, curcumin, and the vehicle was added to $30 \mathrm{~mL}$ of $5 \%$ dextrose solution containing P 407, and then vortexed for 30 seconds. Finally, it was subjected to sonication for 30 minutes using a bath-type sonicator (Cole-Parmer Ultrasonic 8893; Cole-Parmer, Vernon Hills, IL, USA) at $42 \mathrm{KHz}$ to form the LCN dispersion. The LCN dispersion was stored at room temperature for further studies. The compositions of the LCN dispersions are summarized in Table 1.

\section{HPLC}

Curcumin was assayed using an HPLC system equipped with an LC-20AD VP pump (Shimadzu, Japan) and SPD-20A ultraviolet-visible spectroscopy detector at $425 \mathrm{~nm}$. The mobile phase consisted of a mixture of acetonitrile and $2 \%$ acetic acid (65:35\% volume/volume), and the flow rate was set at $0.8 \mathrm{~mL} / \mathrm{minute}$. A C18 Inertsil ODS column (GL Sciences Inc., Tokyo, Japan; $150 \mathrm{~mm} \times 4.6 \mathrm{~mm}, 5 \mu \mathrm{m}$ ) was used, and the injection volume was $20 \mu \mathrm{L}$. HPLC assay validation was performed five times a day for 5 consecutive days at a curcumin concentration range of $0.05-50 \mu \mathrm{g} / \mathrm{mL}$. Rhodamine 6G was used as an internal standard. 
Table I Composition of curcumin-loaded LCN dispersion

\begin{tabular}{lllllll}
\hline Formulation code & MO $(\mathrm{g})$ & PEG $\mathbf{4 0 0}(\mathbf{g})$ & RH 40 $(\mathrm{g})$ & P $407(\mathrm{~g})$ & Curcumin $(\mathrm{g})$ & Water $(\mathbf{m L})$ \\
\hline LCN-P & $\mathrm{I.2}$ & 0.6 & & 0.12 & 0.03 & 30.0 \\
LCN-R & 1.2 & & 0.6 & 0.12 & 0.03 & 30.0 \\
LCN-PR & 1.2 & 0.3 & 0.3 & 0.12 & 0.03 & 30.0 \\
\hline
\end{tabular}

Notes: Three different LCN dispersions were prepared with variations of solubilizer vehicles (ie, PEG 400, RH 40, or a combination of the two). Each value represents the mean $\pm S D(n=3)$.

Abbreviations: MO, monoolein; PEG, polyethylene glycol; RH, Cremophor RH 40; P 407, Poloxamer 407; LCN, liquid crystalline nanoparticle; LCN-P, liquid crystalline nanoparticles prepared with polyethylene glycol 400 as a solubilizer; LCN-R, liquid crystalline nanoparticles prepared with Cremophor RH 40 as a solubilizer; LCN-PR, liquid crystalline nanoparticles prepared with polyethylene glycol 400 and Cremophor RH 40 as solubilizers; SD, standard deviation; n, number.

\section{Characterization of curcumin-loaded LCN dispersion}

Particle size and the polydispersity index (PDI) were determined using a high-performance dynamic light scattering device with Malvern Zetasizer Nano ZS (Malvern Instruments, Malvern, UK) at a fixed angle of $90^{\circ}$. The LCN dispersion was sonicated for 30 seconds and then diluted with distilled water $(50 \mu \mathrm{L} \rightarrow 1.5 \mathrm{~mL})$. The surface charge was measured using an electrophoretic light scattering method at $25^{\circ} \mathrm{C}$. All measurements were performed in triplicate.

The entrapment efficiency (EE) of the LCN dispersion was determined by an ultracentrifugation method. The LCN dispersion $(1 \mathrm{~mL})$ was centrifuged at $2,500 \times g$ for 15 minutes using Amicon ${ }^{\circledR}$ Ultra-4 (molecular weight cut-off: 10,000 g/mol; EMD Millipore, Billerica, MA, USA), and the filtrate containing free curcumin was analyzed using the HPLC system described earlier. Preliminary experiments were performed for the optimization of ultracentrifugation, which proved that $2,500 \times g$ for 15 minutes was sufficient for the separation of the unentrapped drug from the entrapped drug. The total amount of curcumin in the LCN dispersion was measured using HPLC after destruction of the LCN dispersion by dilution with methanol $(\times 20)$. EE was calculated by the following equation:

$$
\mathrm{EE}(\%)=100 \times\left(\mathrm{D}_{\mathrm{t}}-\mathrm{D}_{\mathrm{f}}\right) / \mathrm{D}_{\mathrm{t}},
$$

where $D_{t}$ and $D_{f}$ are the amount of total and free (unentrapped) drug in the LCN dispersion, respectively.

The morphology of the LCN was examined by a transmission electron microscope (TEM) (Hitachi 7600; Hitachi Ltd., Tokyo, Japan). A drop of diluted LCN dispersion was placed in a carbon-coated grid and negatively stained with a $1 \%$ phosphotungstic acid solution followed by air drying. TEM images were taken at an accelerating voltage of $100 \mathrm{kV}$.

The LCN dispersion was put into a vial and stored at two different temperatures $\left(25^{\circ} \mathrm{C}\right.$ and $\left.40^{\circ} \mathrm{C}\right)$. The vials were tightly sealed and covered with aluminum foil to avoid photodegradation of the curcumin. On days 7, 15, 30, and 45, an aliquot of the sample was taken out, and the particle size and surface charge were determined as measures of physical stability of the LCN dispersion. The amount of curcumin remaining in the $\mathrm{LCN}$ dispersion was also determined as a measure of chemical stability of the drug.

The in vitro release of LCN was carried out using the Spectra/Por ${ }^{\circledR}$ dialysis membrane bag. The LCN dispersion $(1 \mathrm{~mL})$ was placed into the dialysis bag (molecular weight cut-off: $3,500 \mathrm{~g} / \mathrm{mole}$ ) and immersed into $10 \mathrm{~mL}$ of release medium (0.01 M phosphate buffer, $\mathrm{pH} 7.4)$ in a falcon tube, with shaking at 50 strokes/minute while maintaining the temperature at $37^{\circ} \mathrm{C}$ using a water bath. At predetermined intervals, the whole medium was withdrawn and replaced with fresh medium to prevent the degradation of curcumin released during the experimental period. The amount of curcumin released was measured by HPLC, as mentioned earlier. At the end of the experiment, the LCN dispersion was withdrawn from the dialysis bag, and the quantity of the remaining curcumin was measured to check the mass balance.

\section{Cellular uptake of curcumin-loaded LCN dispersion}

The cellular uptake of the LCN-R dispersion was studied using fluorescence microscopy and fluorescence-activated cell sorting (FACS) analysis with a human colon cancer cell line (HCT116). For fluorescence microscopy, HCT116 cells were seeded in a six-well plate at a density of $2 \times 10^{4}$ cells per well and incubated for 24 hours to allow attachment. The medium was removed and the cells were washed with Dulbecco's phosphate buffered saline (PBS) and treated with RPMI-1640 medium containing LCN-R, with curcumin concentrations of $1 \mu \mathrm{M}$ and $5 \mu \mathrm{M}$ for 4 hours at $37^{\circ} \mathrm{C}$. After treatment for 4 hours, the cells were rinsed three times with PBS to completely wash out the LCN particles remaining on the surface of the cells. Fresh PBS was added to the plate, and the cells were visualized by fluorescence microscopy (Axio Imager.Z1; Carl Zeiss Meditec AG, Jena, Germany). Curcumin dissolved in dimethyl sulfoxide (DMSO) was also 
tested as a control. For the FACS analysis, the sample preparation was identical to the fluorescence microscopy study, except for the subsequent trypsinization step for FACS. After trypsinization, the detached cells were collected into a FACS tube with the culture medium, and fluorescence intensity was analyzed at an excitation wavelength of $488 \mathrm{~nm}$ using a band pass filter (530/30) in FACSCalibur ${ }^{\mathrm{TM}}$ flow cytometer (BD Biosciences, San Jose, CA, USA).

\section{Anticancer activity of curcumin-loaded LCN dispersion}

The anticancer activity of curcumin-loaded LCN was assessed using the 3-(4,5-dimethylthiazol-2-yl)-2,5-diphenyltetrazolium bromide (MTT) assay on the human colon cancer cell line (HCT116). The cells were seeded in a 96-well plate at a density of $4 \times 10^{4}$ cells per well in RPMI-1640 medium with $10 \%$ fetal bovine serum, and they were incubated for 24 hours to allow attachment. Subsequently, the medium was replaced with fresh medium containing LCN dispersion with curcumin concentrations of $1 \mu \mathrm{M}, 5 \mu \mathrm{M}, 10 \mu \mathrm{M}$, and $20 \mu \mathrm{M}$ by serial dilution. After 4 hours, the medium containing the nanoparticles was removed and $100 \mu \mathrm{L}$ of the MTT solution $(0.5 \mathrm{mg} / \mathrm{mL})$ was added to each well; the cells were then placed in a humidified incubator $\left(37^{\circ} \mathrm{C}, 5 \% \mathrm{CO}_{2}\right)$ for 4 hours. Then, $100 \mu \mathrm{L}$ of DMSO was added and the cells were left in a dark area at room temperature. The plate was shaken for 5 minutes on a plate shaker, and the absorbance was measured at $570 \mathrm{~nm}$ using a microplate reader. Curcumin dissolved in DMSO was also tested. Cell growth inhibition was calculated by the following equation:

$$
\text { Cell growth inhibition }(\%)=100 \times\left(\mathrm{A}_{0}-\mathrm{A}_{\mathrm{c}}\right) / \mathrm{A}_{0} \text {, }
$$

where $A_{c}$ is the absorbance of the cells treated with the LCN-R dispersion and $A_{0}$ is the absorbance of the cells treated only with the medium. All experiments were repeated six times.

\section{Effect of the curcumin-loaded LCN dispersion on cell cycle distribution}

To investigate the effect of curcumin on the cell cycle distribution, the HCT116 cell line and the FACSCalibur flow cytometer were used. The cells were seeded in a six-well plate $\left(2 \times 10^{4}\right.$ cells per well) and incubated for 24 hours. The medium was replaced and the cells were incubated with the LCN-R dispersion at $37^{\circ} \mathrm{C}$. After 4 hours, the medium was removed, and the cells were washed twice with fresh PBS and collected by trypsinization. The cells were then suspended in $50 \mu \mathrm{L}$ of an ice-cold PBS buffer solution. After fixation in ice-cold methanol for 1 hour at $4{ }^{\circ} \mathrm{C}$, the supernatant was discarded after centrifugation. The cells were washed twice and finally incubated in PBS containing ribonuclease for 30 minutes at $37^{\circ} \mathrm{C}$. Cells were then chilled on ice for 10 minutes and stained with propidium iodide $(50 \mu \mathrm{g} / \mathrm{mL})$ for 30 minutes. The cell cycle distribution was studied using 10,000 cells per analysis using a FACSCalibur flow cytometer. Data were analyzed using CellQuest ${ }^{\mathrm{TM}}$ software (BD Biosciences).

\section{Statistical analysis}

All of the data obtained were analyzed by Student's $t$-test except for the results for the cell cycle distribution study. The data were presented as the mean \pm standard deviation. A value of $P<0.05$ was considered statistically significant.

\section{Results}

Since curcumin is not soluble in MO, we performed solubility tests to choose the vehicles to solubilize curcumin. The solubility test results showed that curcumin had the highest solubility in PEG $400(146.26 \pm 7.8 \mathrm{mg} / \mathrm{mL})$ followed by RH $40(41.47 \pm 0.74 \mathrm{mg} / \mathrm{mL})$. Therefore, we used the two vehicles to solubilize curcumin and prepare the LCN dispersions of the drug. Figure 2 shows the solubility results of curcumin in the different vehicles examined.

Three different LCN dispersions were prepared by a sonication method using PEG 400 (LCN-P), RH 40 (LCN-R), and a combination of the two vehicles (LCN-PR) as solubilizers of the drug. The mean particle sizes were $271.90 \pm 3.77 \mathrm{~nm}$, $233.93 \pm 1.66 \mathrm{~nm}$, and $251.43 \pm 1.08 \mathrm{~nm}$ for LCN-P, LCN-R,

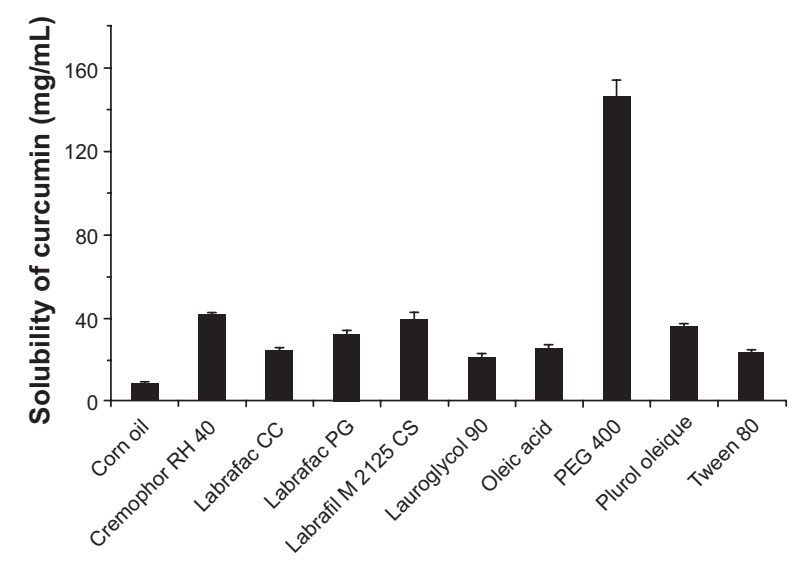

Figure 2 Solubility of curcumin in various vehicles.

Notes: An excess amount of curcumin was mixed with each vehicle and shaken in a water bath maintaining temperature at $37^{\circ} \mathrm{C}$ for 24 hours. Each value represents the mean \pm standard deviation (number $=3$ ).

Abbreviation: PEG, polyethylene glycol. 
Table 2 Characterization of curcumin-loaded LCN dispersion

\begin{tabular}{lllll}
\hline $\begin{array}{l}\text { Formulation } \\
\text { code }\end{array}$ & Particle size $(\mathbf{n m})$ & $\begin{array}{l}\text { Surface charge } \\
\text { (zeta potential, } \mathbf{m V})\end{array}$ & EE (\%) & Color \\
\hline LCN-P & $271.90 \pm 3.77^{*, a}$ & $-22.58 \pm 1.38$ & $99.97 \pm 0.01$ & Yellow \\
LCN-R & $233.93 \pm 1.66^{\circ}$ & $-19.86 \pm 0.24$ & $99.96 \pm 0.01$ & Yellow \\
LCN-PR & $251.43 \pm 1.08$ & $-22.33 \pm 0.87$ & $99.98 \pm 0.02$ & Yellow \\
\hline
\end{tabular}

Notes: Each value represents the mean $\pm S D(n=3)$. $* P<0.001$ compared to $L C N-R ;$ a $P<0.05$ compared to $L C N-P R$; ${ }^{b} P<0.00$ I compared to $L C N-P R$.

Abbreviations: LCN, liquid crystalline nanoparticles; EE, entrapment efficiency; LCN-P, liquid crystalline nanoparticles prepared with polyethylene glycol 400 as a solubilizer; LCN-R, liquid crystalline nanoparticles prepared with Cremophor RH 40 as a solubilizer; LCN-PR, liquid crystalline nanoparticles prepared with polyethylene glycol 400 and Cremophor RH 40 as solubilizers; SD, standard deviation; n, number.

and LCN-PR, respectively (Table 2). The PDI was less than 0.3 , as measured by dynamic light scattering, indicating the homogeneous particle size distribution in all formulations. There was a significant difference in particle size among the LCN dispersions prepared with the three different compositions. The EE of curcumin into the LCN was very high, and this was independent of the composition used in this study. For all three LCN formulations prepared with different solubilizers, the EE was as high as $99 \%$ or above, as shown in Table 2.
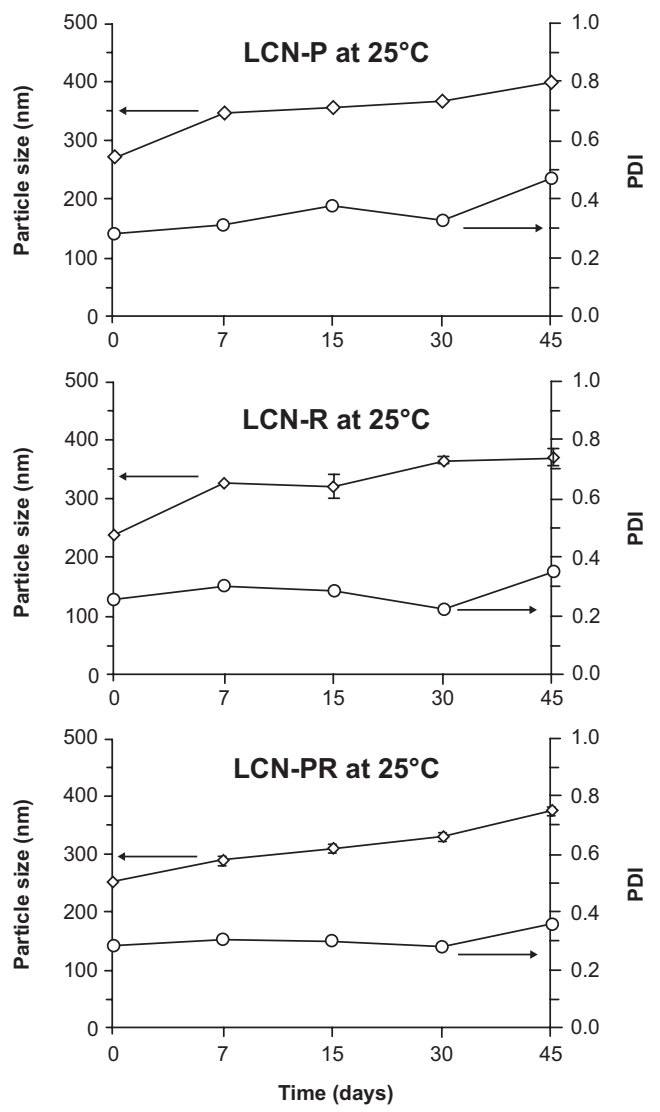

During the stability study, the mean particle size of the LCN dispersion was slightly increased in all formulations, but the size evolution was not significant (Figure 3). Accordingly, the PDI also increased slightly. The surface charge of the LCN dispersion did not significantly change over the 45 days of the stability study period, even at $40^{\circ} \mathrm{C}$ (Figure 4). The amount of curcumin remaining after a 15-day storage period at $25^{\circ} \mathrm{C}$ was $>90 \%$ in LCN-P, while it was $<90 \%$ in the other two formulations (LCN-R and LCN-PR). Even at an elevated temperature of $40^{\circ} \mathrm{C}$ for 45 days, it was $75.26 \% \pm 2.64 \%$,
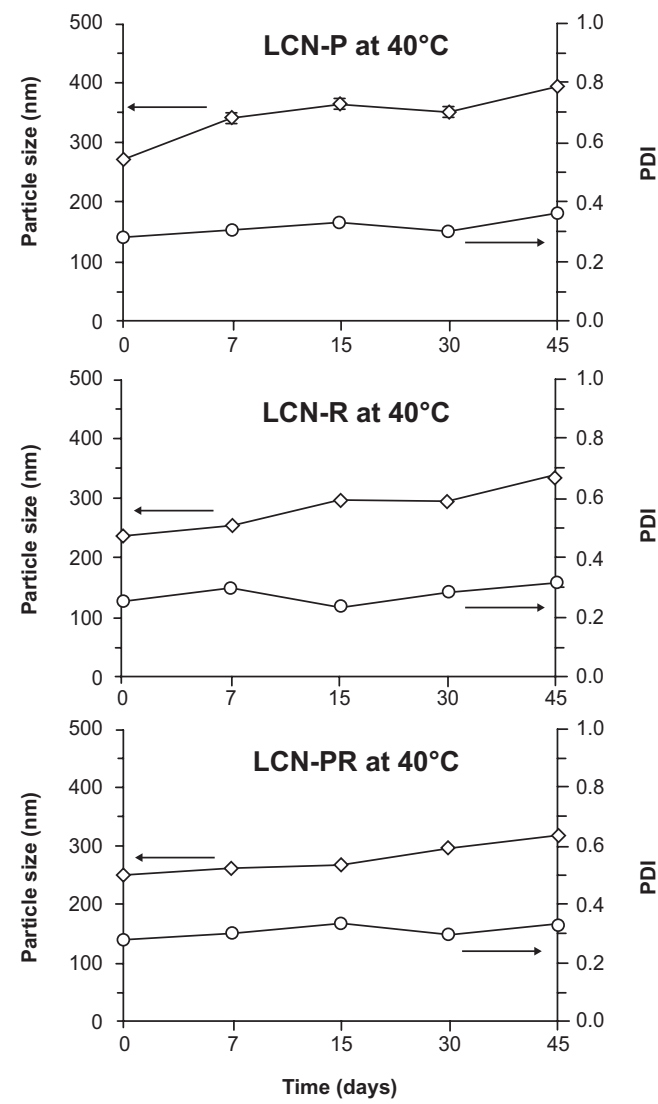

Figure 3 Particle size evolution of curcumin-loaded LCN dispersions at $25^{\circ} \mathrm{C}$ and $40^{\circ} \mathrm{C}$.

Notes: Three different LCN dispersions were stored at $25^{\circ} \mathrm{C}$ and $40^{\circ} \mathrm{C}$, and the particle size evolution was determined for 45 days as a measure of physical stability. Each value represents the mean \pm standard deviation (number=3). Arrows indicate $Y$ axis value corresponding to particle size/PDI.

Abbreviations: LCN, liquid crystalline nanoparticles; LCN-P, liquid crystalline nanoparticles prepared with polyethylene glycol 400 as a solubilizer; PDI, polydispersity index; LCN-R, liquid crystalline nanoparticles prepared with Cremophor RH 40 as a solubilizer; LCN-PR, liquid crystalline nanoparticles prepared with polyethylene glycol 400 and Cremophor RH 40 as solubilizers. 

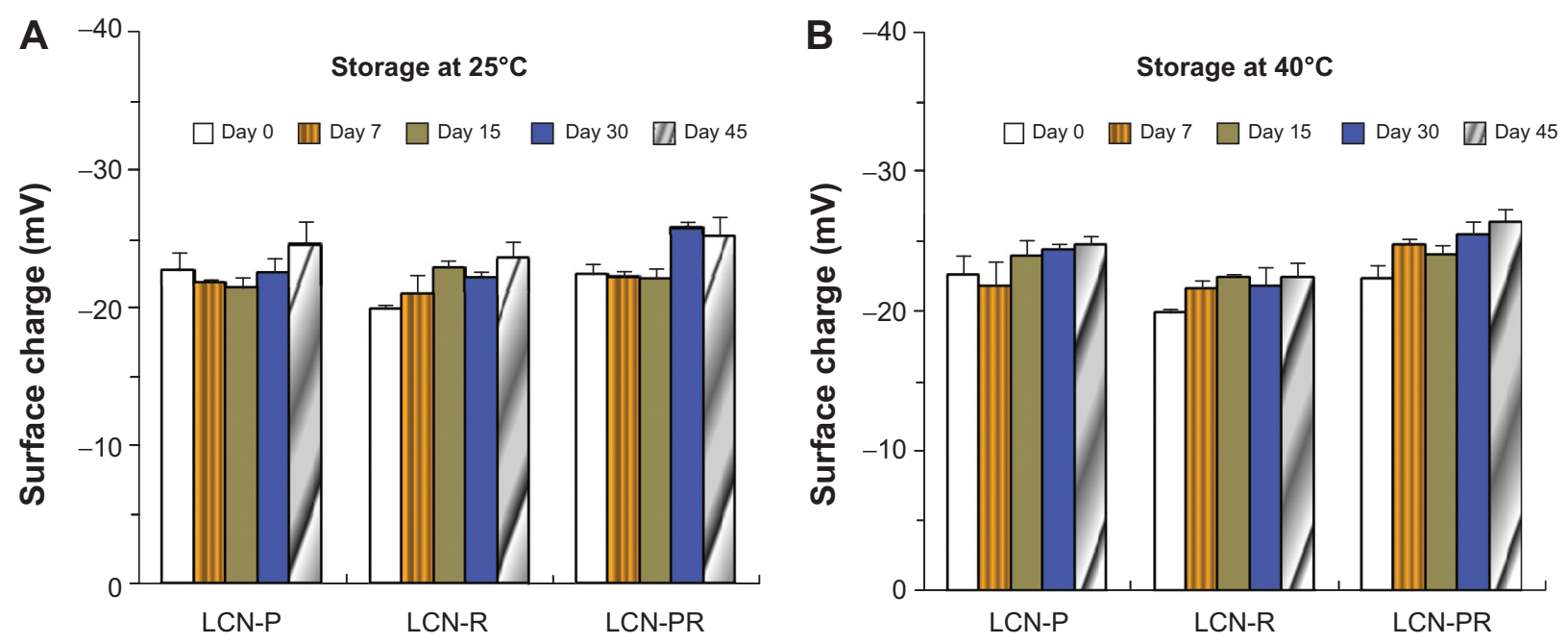

Figure 4 Surface charge evolution of curcumin-loaded LCN dispersions at $25^{\circ} \mathrm{C}$ and $40^{\circ} \mathrm{C}$

Notes: Storage at $(\mathbf{A}) 25^{\circ} \mathrm{C}$ and $(B) 40^{\circ} \mathrm{C}$. Each value represents the mean \pm standard deviation $(n=3)$.

Abbreviations: LCN, liquid crystalline nanoparticles; LCN-P, liquid crystalline nanoparticles prepared with polyethylene glycol 400 as a solubilizer; LCN-R, liquid crystalline nanoparticles prepared with Cremophor RH 40 as a solubilizer; LCN-PR, liquid crystalline nanoparticles prepared with polyethylene glycol 400 and $\mathrm{Cremophor} \mathrm{RH} 40$ as solubilizers; n, number.

$73.57 \% \pm 8.00 \%$, and $77.03 \% \pm 9.58 \%$ for LCN-P, LCN-R, and LCN-PR, respectively (Figure 5), while 100\% of curcumin was degraded when the drug was dissolved in methanol and stored at the same temperature (data not shown).
The TEM images revealed discrete and monodispersed nanoparticles without aggregation (Figure 6). The morphology of the particles exhibited a multifaceted geometry, mostly being hexagonal, and the particle size was $<300 \mathrm{~nm}$,

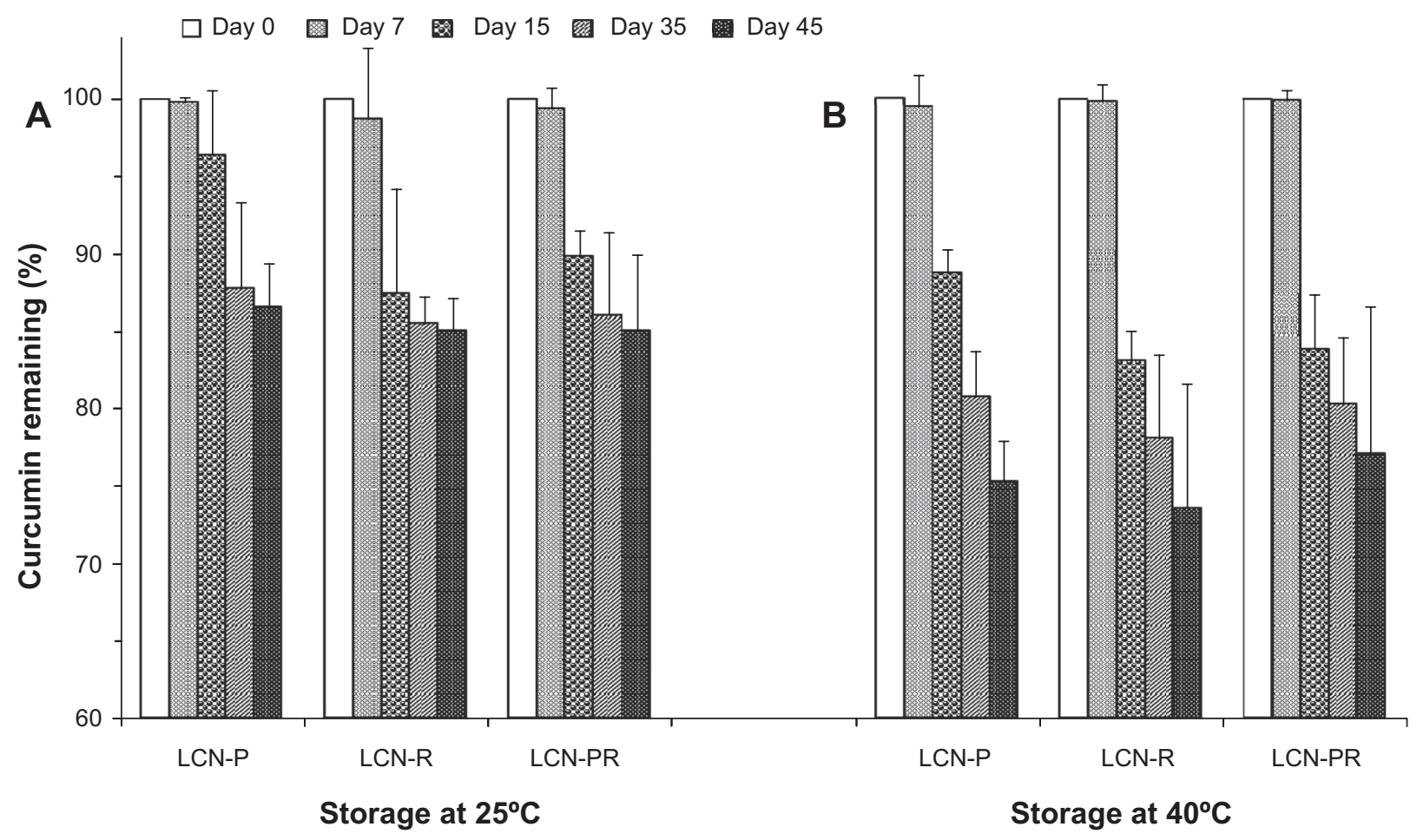

Figure 5 Chemical stability of curcumin-loaded LCN dispersions over 45 days at $25^{\circ} \mathrm{C}$ and $40^{\circ} \mathrm{C}$.

Notes: Three different LCN dispersions were stored at $(\mathbf{A}) 25^{\circ} \mathrm{C}$ and $(\mathbf{B}) 40^{\circ} \mathrm{C}$, and the amount of curcumin was determined for 45 days as a measure of chemical stability. Each value represents the mean \pm standard deviation $(n=3)$.

Abbreviations: LCN, liquid crystalline nanoparticles; LCN-P, liquid crystalline nanoparticles prepared with polyethylene glycol 400 as a solubilizer; LCN-R, liquid crystalline nanoparticles prepared with Cremophor RH 40 as a solubilizer; LCN-PR, liquid crystalline nanoparticles prepared with polyethylene glycol 400 and $\mathrm{Cremophor} \mathrm{RH} 40$ as solubilizers; n, number. 

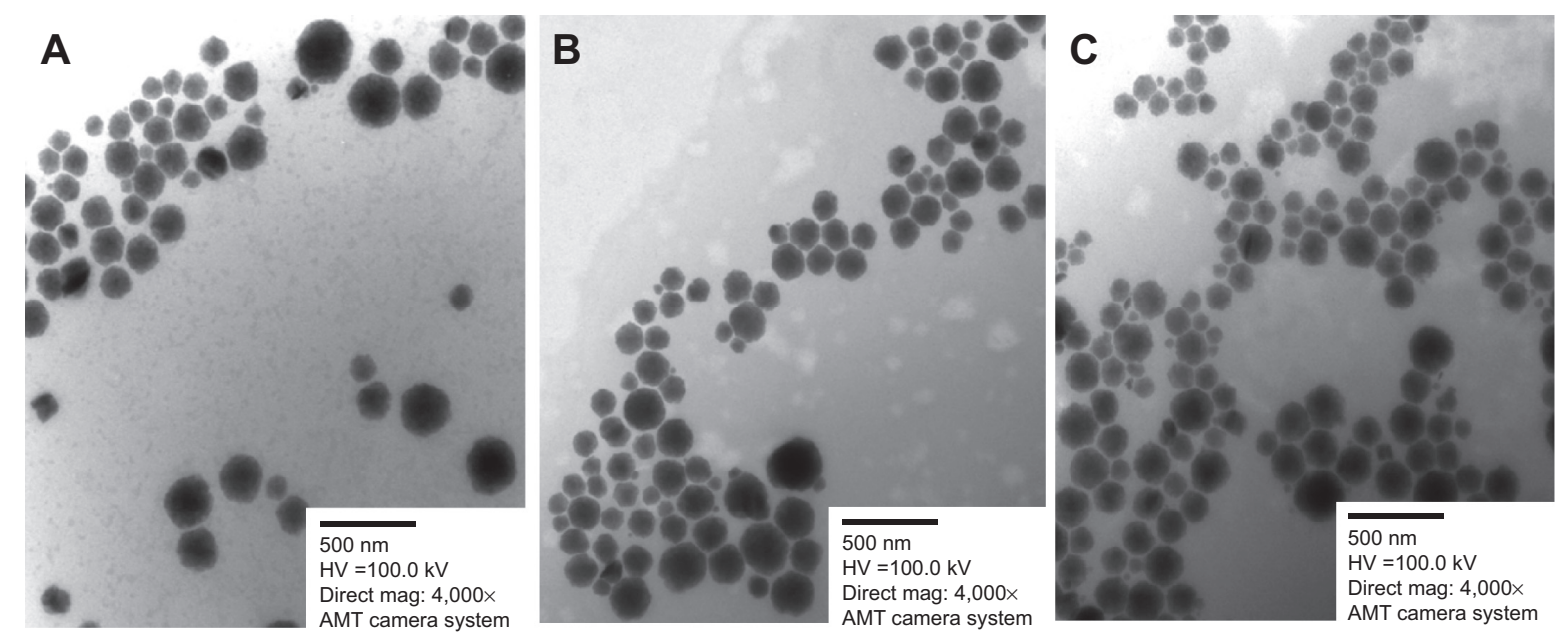

Figure 6 TEM images of curcumin-loaded LCN dispersions $(\mathrm{bar}=500 \mathrm{~nm})$.

Notes: (A) LCN-P; (B) LCN-R; (C) LCN-PR. Morphology of the three different LCN dispersions was examined by TEM and it revealed discrete, monodispersed, multifaceted (mostly hexagonal) nanoparticles.

Abbreviations: TEM, transmission electron microscope; LCN, liquid crystalline nanoparticles; LCN-P, liquid crystalline nanoparticles prepared with polyethylene glycol 400 as a solubilizer; LCN-R, liquid crystalline nanoparticles prepared with Cremophor RH 40 as a solubilizer; LCN-PR, liquid crystalline nanoparticles prepared with polyethylene glycol 400 and Cremophor RH 40 as solubilizers.

which is consistent with the dynamic light scattering characterization.

Figure 7 illustrates the in vitro release profile of curcumin from the LCN dispersions prepared with different solubilizers. LCN prepared with PEG 400 (LCN-P) exhibited the slowest release of curcumin, showing that only $2.04 \%$ was released and $77.44 \%$ remained in the LCN at the end of the

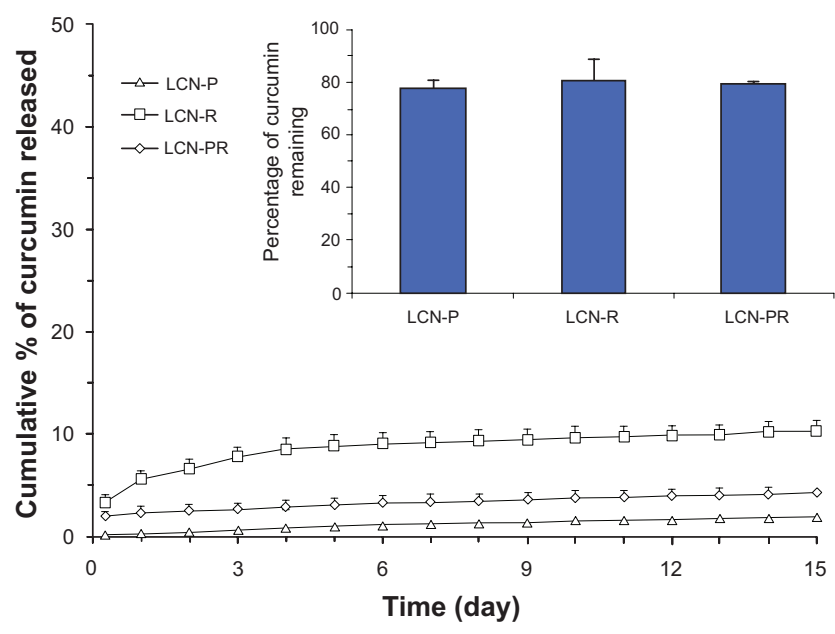

Figure 7 Cumulative in vitro release profile of curcumin from LCN dispersions in phosphate-buffered saline $\left(\mathrm{pH} \mathrm{7.4)}\right.$ at $37^{\circ} \mathrm{C}$.

Notes: Inset: percentage of curcumin remaining within the LCN at the end of the release study. The in vitro release of the three curcumin-loaded LCN dispersions was carried out using a dialysis bag (molecular weight cut-off: 3,500 g/mole), and it revealed a sustained release pattern, especially for LCN-P. Each value represents the mean \pm standard deviation $(n=3)$.

Abbreviations: LCN, liquid crystalline nanoparticles; LCN-P, liquid crystalline nanoparticles prepared with polyethylene glycol 400 as a solubilizer; LCN-R, liquid crystalline nanoparticles prepared with Cremophor RH 40 as a solubilizer; LCNPR, liquid crystalline nanoparticles prepared with polyethylene glycol 400 and Cremophor RH 40 as solubilizers; n, number. release study ( $20.52 \%$ degraded). Although LCN-R showed the fastest release, only $10.32 \%$ of curcumin was released and $80.08 \%$ remained in the LCN (9.6\% degraded). The release of curcumin from the LCN-PR was intermediate (4.25\%) between the other two dispersions.

To investigate the cellular uptake of LCN-R, fluorescence microscopy and FACS analysis were used with the curcumin molecule as a fluorescence marker (yellowish green). Figure $8 \mathrm{~A}$ shows that the uptake of LCN-R by the HCT116 cells was dose-dependent, whereas the drug dissolved in DMSO was not. This was further evidenced by FACS analysis, showing $81.45 \%$ gating in curcumin $1 \mu \mathrm{M}$ as LCN-R, which is $80.09 \%$ greater than the drug dissolved in DMSO at the same concentration (1.36\%; Figure $8 \mathrm{~B})$. When the drug was treated as LCN-R $(5 \mu \mathrm{M})$, the fluorescence gating was $99.1 \%$.

Figure 9 depicts the cell growth inhibition of curcumin as a measure of anticancer activity at different concentrations of the drug dissolved in DMSO and as an LCN dispersion. Clearly, the LCN-R dispersion exhibited growth inhibition of HCT116 cells in a dose-proportional manner, while the drug dissolved in DMSO did not. Cell growth inhibition of LCN-P, LCN-R, and LCN-PR at $20 \mu \mathrm{M}$ was $71.74 \%, 70.37 \%$, and $64.17 \%$, respectively, and there was no significant difference among the formulations.

Figure 10 shows the effect of curcumin treatment on the cell cycle distribution of HCT116 cells at different concentrations of the drug dissolved in DMSO and as LCN-R. Since there was no significant difference identified among 


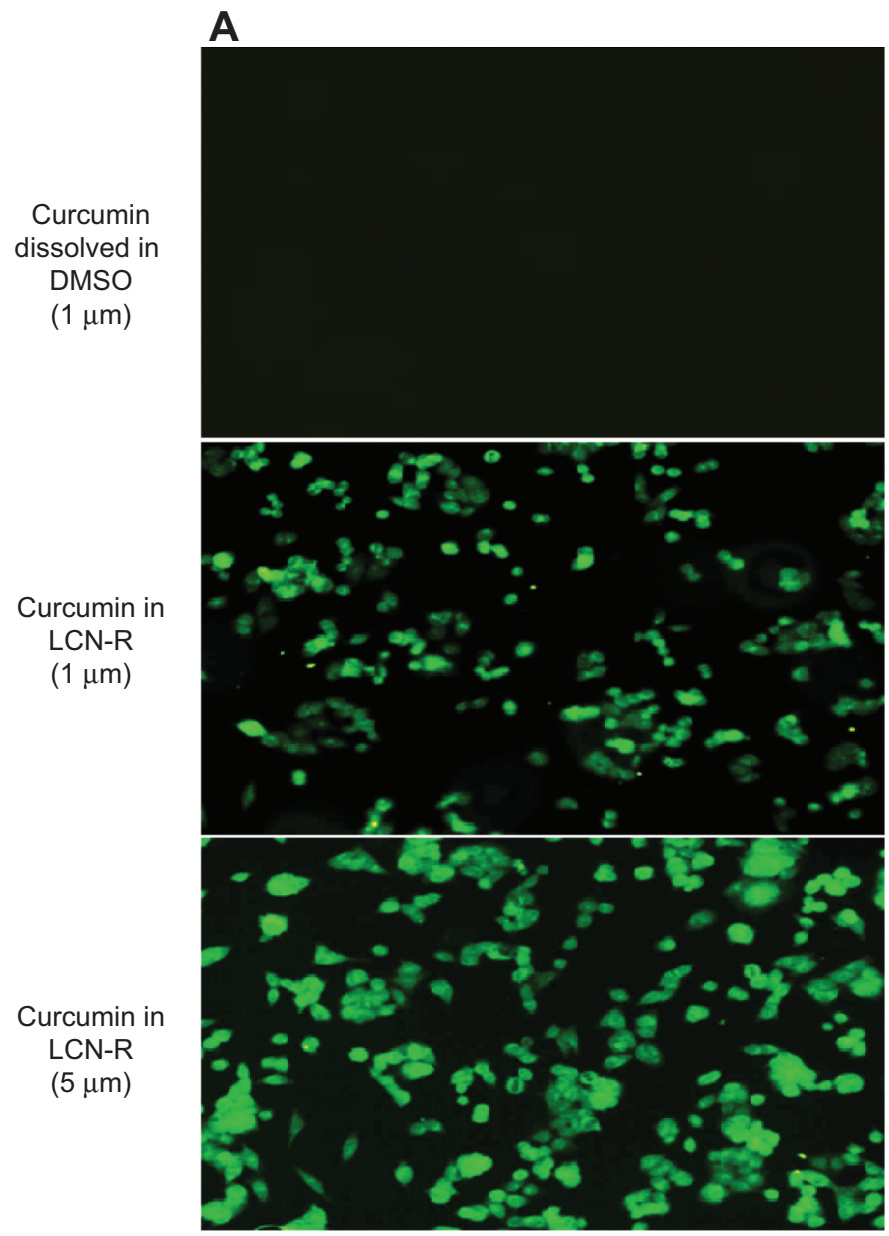

B
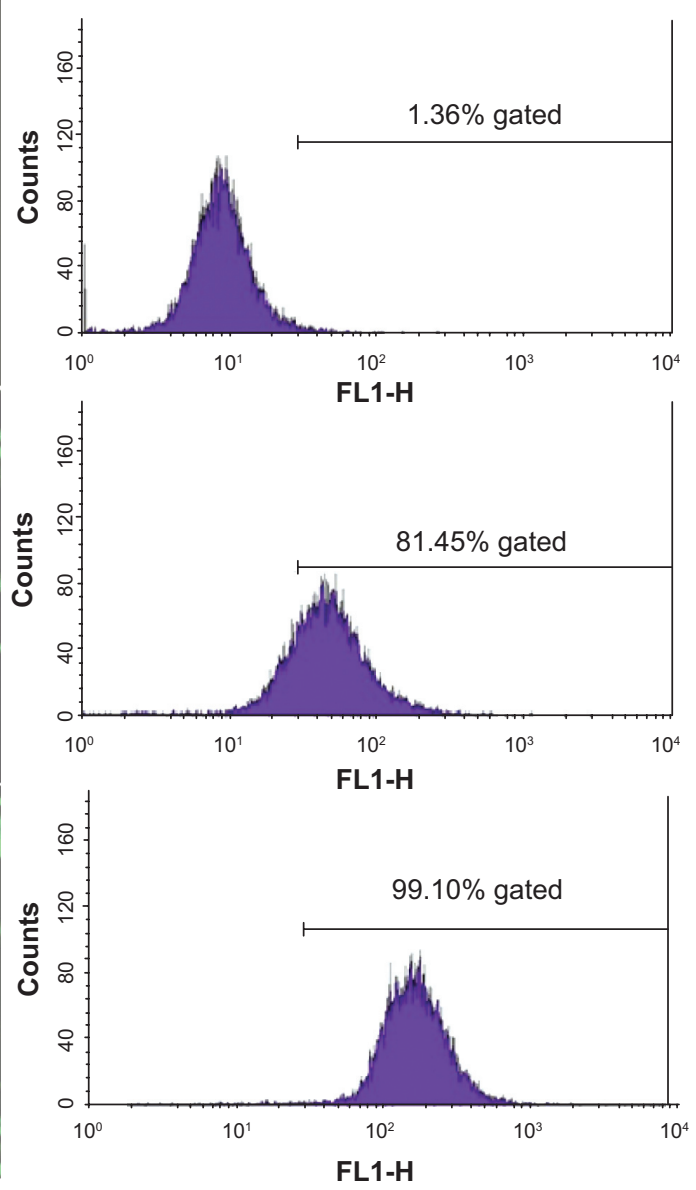

Figure 8 Cellular uptake of a curcumin-loaded LCN dispersion (LCN-R) using a human colon cancer cell line (HCTII6).

Notes: (A) Fluorescence microscopy image; (B) FACS analysis. The cellular uptake of the curcumin-loaded LCN dispersion was studied using fluorescence microscopy and FACS analysis with a human colon cancer cell line. The uptake of curcumin-loaded LCN by the HCTII 6 cells occurred in a dose-dependent manner.

Abbreviations: DMSO, dimethyl sulfoxide; FLI-H, fluorescence channel I height; LCN-R, liquid crystalline nanoparticles prepared with Cremophor RH 40 as a solubilizer; LCN, liquid crystalline nanoparticles; FACS, fluorescence-activated cell sorting.

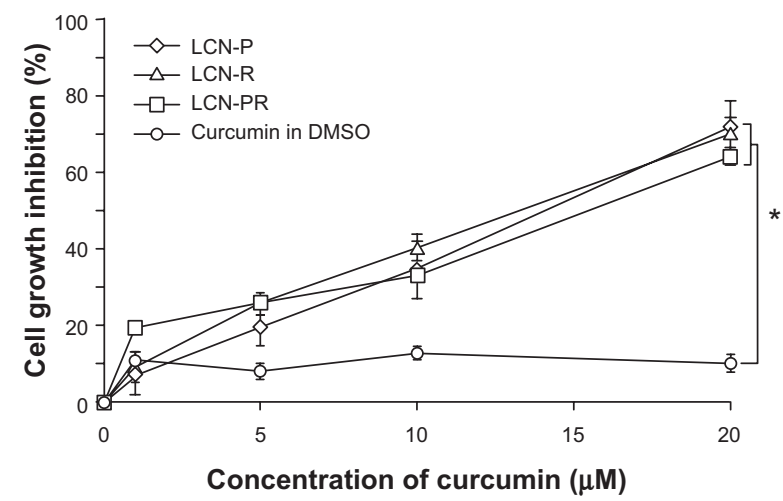

Figure 9 Cell growth inhibition of a human colon cancer cell line (HCTII6) by curcumin dissolved in DMSO and as LCN dispersions.

Notes: Anticancer activity of curcumin-loaded LCN was investigated with a human colon cancer cell line. All the three LCN dispersions exhibited dose-dependent activity, and the cell growth inhibition was significantly higher than the drug dissolved in DMSO. Each value represents the mean \pm standard deviation $(n=6) . * P<0.00$ l.

Abbreviations: LCN, liquid crystalline nanoparticles; LCN-P, liquid crystalline nanoparticles prepared with polyethylene glycol 400 as a solubilizer; LCN-R, liquid crystalline nanoparticles prepared with Cremophor RH 40 as a solubilizer; LCN$\mathrm{PR}$, liquid crystalline nanoparticles prepared with polyethylene glycol 400 and Cremophor RH 40 as solubilizers; DMSO, dimethyl sulfoxide; n, number. the LCN dispersions in the cell growth inhibition study, only LCN-R was examined in this study. Curcumin-induced DNA degradation occurred in a dose-dependent manner when the cells were treated with the drug as an LCN dispersion. The percentage of sub-G0 population (apoptosis) reached 5.92\% and $53.09 \%$ at $5 \mu \mathrm{M}$ and $10 \mu \mathrm{M}$ of curcumin entrapped in the LCN-R, respectively. In contrast, curcumin dissolved in DMSO did not show any significant change in the cell cycle distribution up to $5 \mu \mathrm{M}$, and it did not induce noticeable cell death. Instead, it caused G2/M arrest of the cells beginning with $10 \mu \mathrm{M}$ of the drug, which does not indicate cell death. Cell death caused by LCN-R was 8.6-fold compared to that caused by the drug dissolved in DMSO.

\section{Discussion}

Curcumin is a potential therapeutic agent for cancer treatment. Despite its promising pharmacological efficacy and safety, the clinical use of the drug has been limited, owing 

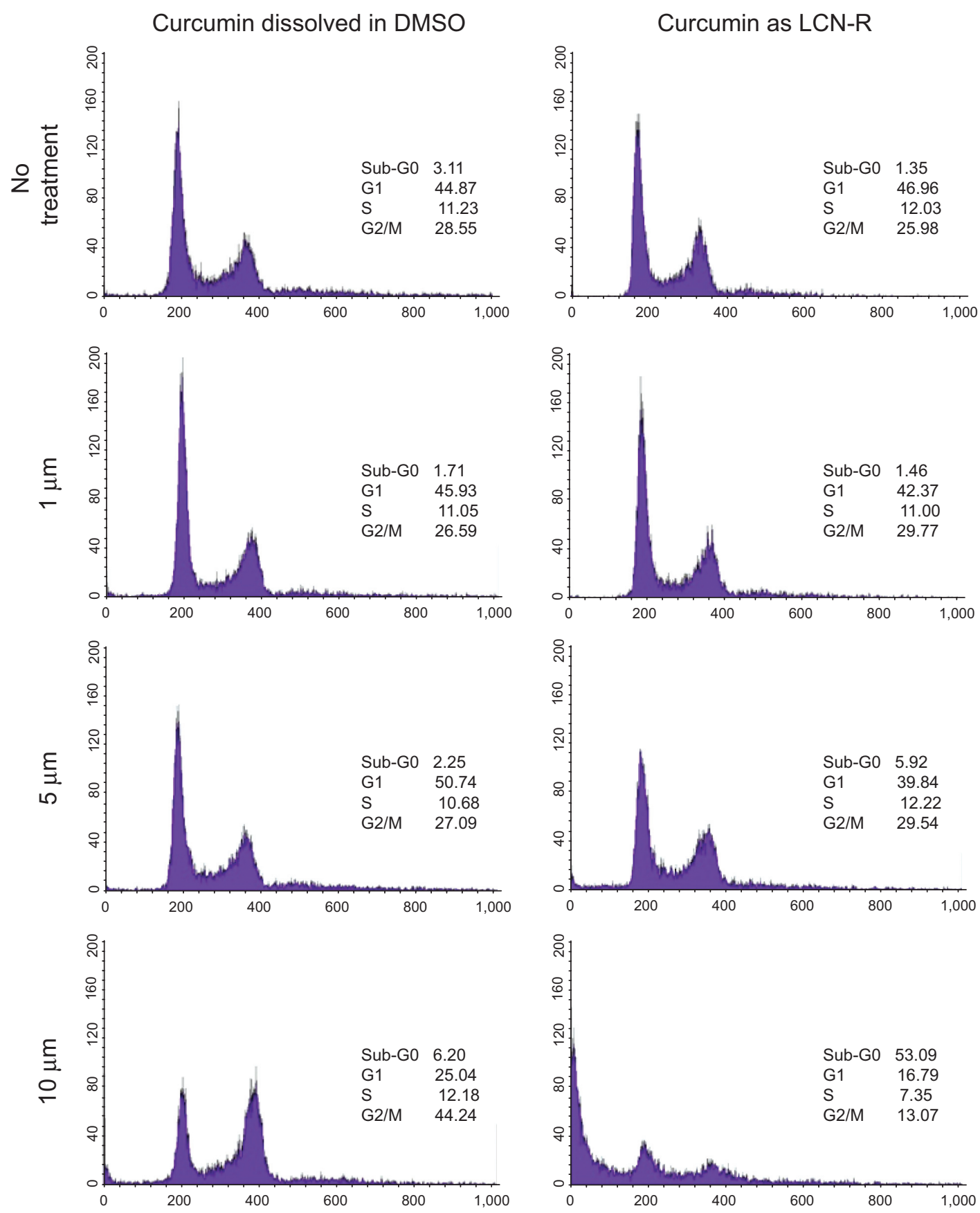

Figure 10 The effect of curcumin on the cell cycle distribution of a human colon cancer cell line (HCTII6).

Notes: The effect of curcumin-loaded LCN dispersion was investigated with a human colon cancer cell line using FACS analysis. Curcumin induced apoptosis of the cells in a dose-dependent manner when the cells were treated with the drug as an LCN dispersion. The percentage of the sub-G0 population reached $53.09 \%$ at $10 \mu \mathrm{m}$ of curcumin entrapped in the LCN.

Abbreviations: DMSO, dimethyl sulfoxide; LCN-R, liquid crystalline nanoparticle dispersion prepared with Cremophor RH 40 as a solubilizer; LCN, liquid crystalline nanoparticles; FACS, fluorescence-activated cell sorting.

to its poor solubility, extremely low bioavailability, short half-life, and chemical fragility. ${ }^{4}$ To overcome these limitations, pharmaceutical formulations using nanoparticle-based drug delivery systems have been widely studied; however, development of an effective drug delivery system of curcumin for use as a chemotherapeutic agent has remained a challenge. ${ }^{23}$
We have successfully prepared a water-dispersible formulation of curcumin using an MO-based LCN technology by modifying the process - that is, dissolving the drug into a solubilizer at the beginning of the preparation procedure, followed by the classic method of preparation of LCN. PEG 400 and RH 40 were chosen as solubilizers because they revealed the best results among all the candidate solubilizers examined. It 
appears that oxygen atoms in the ethylene oxide moiety in PEG 400 and RH 40 formed hydrogen binding with protons in the three hydroxyl groups of the curcumin molecule.

As far as bioavailability is concerned, the consensus is that the gastrointestinal absorption of curcumin is extremely low. ${ }^{24,25}$ Sharma et $\mathrm{al}^{25}$ reported that neither curcumin nor its metabolites (curcumin glucuronide, curcumin sulfate, hexahydrocurcumin, or hexahydrocurcuminol) were found in the plasma or urine after up to 29 days of oral daily dose administration with 36-180 mg of curcumin in patients with colorectal cancer. Studies have reported earlier that this is because of its low bioavailability and short half-life, which is associated with poor absorption, ${ }^{24,26}$ metabolism at the gut microsome, ${ }^{27,28}$ and first-pass effects in the liver. ${ }^{29}$ The rapid metabolism of curcumin at intestinal and liver microsomes, coupled with poor absorption, appears to be an insurmountable obstacle for the formulation of an oral route of administration. Therefore, we designed our formulation to fit to parenteral administration using injectable solubilizer molecules and 5\% dextrose water as a diluent of the MO-based LCN dispersion. MO, PEG 400, P 407, and RH 40 are generally recognized as safe for pharmaceutical applications.

It is widely known that curcumin undergoes rapid hydrolytic degradation, so $<10 \%$ of the drug can survive within 1 hour in an aqueous solution with a $\mathrm{pH}>7.0$. In neutral and alkaline conditions, curcumin degrades to feruloyl methane and ferulic acid, which are fragments of the parent molecule. ${ }^{30,31}$ Notably, in our study, curcumin entrapped into our LCN formulation was stable, showing that about $75 \%$ remained, even at the end of the stability study (at 45 days) at $40^{\circ} \mathrm{C}$. This result is in good agreement with other researchers' findings, as reported by Esposito et al. ${ }^{18}$ The reason for this remarkable stability enhancement may be due to curcumin molecules being tightly entrapped inside the bicontinuous channel of the crystalline phase of the LCN, which renders protection against an environmental attack. It is also conceivable that water molecules entrapped in the channel of the crystalline phase cannot be mobilized for hydrolytic degradation of the drug. The weak acidic $\mathrm{pH}$ (5.4) of our LCN dispersion might have also contributed to stability enhancement.

The EE of curcumin into the LCN dispersion was almost $100 \%$, irrespective of the composition of the formulations examined in this study, which is similar to the results of our previous experiments with other hydrophobic drugs such as finasteride and tacrolimus (Table 2). ${ }^{19-21,32}$ In our earlier studies conducted with hydrophobic drugs, the EE was also close to $100 \%$, indicating nonspecific interactions between the drug molecule and the hydrophobic moiety of $\mathrm{MO}$ - notably, hydrophobic binding and a van der Waals interaction.

The in vitro release profile of curcumin from the LCN dispersion was sustained over 15 days (Figure 7). A burst effect was observed during the first day. The burst release amount was $<5 \%$ of the drug entrapped in the LCN formulation prepared with PEG 400 (LCN-P, LCN-PR) as a solubilizer molecule. However, the burst release in LCN-R was $>5 \%$ but $<10 \%$ of the drug entrapped. This finding suggests that the ratio of the two solubilizers could be tailored depending on how much burst release is needed. The weight of the solubilizer used in this study was $50 \%$ with respect to $\mathrm{MO}$, but variations in the ratio might also be necessary to meet individual requirements.

The cellular uptake study revealed that the uptake of curcumin was better when HCT116 cells were treated with LCN, as compared to when the cells were treated with curcumin dissolved in DMSO (Figure 8). This finding indicates that curcumin was not internalized by passive diffusion, but by an active cellular mechanism such as endocytosis (Figure 8). Consistent with the findings of other studies, ${ }^{33,34}$ the fluorescence intensity inside the cell was uniform and even throughout the perinuclear region of the cells. Furthermore, FACS analysis showed that the fluorescence intensity rendered by intracellular uptake was proportional to the concentration of curcumin.

We evaluated the effect of the LCN dispersion on anticancer activity and cell cycle distribution using an HCT116 cell line. Cell growth inhibition was significantly higher when the cells were treated with curcumin as LCN, as compared to when the cells were treated with curcumin dissolved in DMSO $(P<0.001)$. This difference may be because the LCN was endocytosed across the cell membrane and diffused throughout the cytoplasm, whereas curcumin itself stays in the cell membrane because it is a liposoluble molecule that is easily incorporated into the cell membrane. ${ }^{35,36}$ In this study, the curcumin treatment time was 4 hours, which is a short time for the drug molecules to permeate into the cell membrane and exert anticancer effects. We chose 4 hours because we were interested in finding anticancer effects rendered by endocytosis of the LCN-R (not the effect rendered by permeation into the cancer cell). Once LCN is taken up, the liquid crystalline structure will be disrupted by lipases in the cytoplasm, and curcumin will be released from the $\mathrm{LCN}$, thereby expressing anticancer activity - that is, the inhibition of cell proliferation and apoptosis induction. ${ }^{37,38}$ This feasibility is supported by the results demonstrated by a cell cycle study, showing a sub-G0 population (apoptosis) of $53.09 \%$ at $10 \mu \mathrm{M}$ of curcumin entrapped in the LCN-R compared to $6.20 \%$ in the control. Based on the present study results, it appears that an MO-based 
LCN dispersion may be a promising carrier of curcumin, so it can be delivered into cancer cells via intravenous administration or direct injection to a tumor mass. Further research is warranted to substantiate the therapeutic application of the MO-based LCN for the treatment of colon cancer, together with the mechanism behind apoptosis induction. One limitation of this study is that the toxicity control of LCN-R against noncancerous cells needs to be performed to prove the specificity of LCN on the apoptosis of malignant cell lines.

\section{Conclusion}

In conclusion, curcumin was successfully entrapped into an MO-based LCN with almost 100\% EE. The LCN dispersion was very stable in terms of its size and surface charge evolution, and it revealed the sustained release of curcumin. In addition, the LCN was efficiently taken up by the HCT116 cell line, and it exhibited markedly enhanced anticancer activity - that is, the inhibition of cell proliferation and apoptosis induction. This finding suggests that the entrapment of curcumin into an MO-based LCN may provide a strategy through which to overcome the hurdles associated with both stability and cellular uptake issues of the drug for the treatment of various cancers in the future.

\section{Acknowledgment}

This work was supported by the Gachon University research fund of 2014 (GCU 2014-M001).

\section{Disclosure}

The authors report no conflicts of interest in this work.

\section{References}

1. Peer D, Karp JM, Hong S, Farokhzad OC, Margalit R, Langer R. Nanocarriers as an emerging platform for cancer therapy. Nat Nanotechnol. 2007;2(12):751-760.

2. Choudhuri T, Pal S, Das T, Sa G. Curcumin selectively induces apoptosis in deregulated cyclin D1-expressed cells at G2 phase of cell cycle in a p53-dependent manner. J Biol Chem. 2005;280(20): 20059-20068.

3. Ravindran J, Prasad S, Aggarwal BB. Curcumin and cancer cells: how many ways can curry kill tumor cells selectively? AAPS J. 2009;11(3): 495-510.

4. Wilken R, Veena MS, Wang MB, Srivatsan ES. Curcumin: A review of anti-cancer properties and therapeutic activity in head and neck squamous cell carcinoma. Mol Cancer. 2011;10-12.

5. Vyas A, Dandawate P, Padhye S, Ahmad A, Sarkar F. Perspectives on new synthetic curcumin analogs and their potential anticancer properties. Curr Pharm Des. 2013;19(11):2047-2069.

6. Syng-Ai C, Kumari AL, Khar A. Effect of curcumin on normal and tumor cells: role of glutathione and bcl-2. Mol Cancer Ther. 2004;3(9): 1101-1108.

7. Agarwal B, Swaroop P, Protiva P, Raj SV, Shirin H, Holt PR. Cox-2 is needed but not sufficient for apoptosis induced by Cox-2 selective inhibitors in colon cancer cells. Apoptosis. 2003;8(6):649-654.
8. Azuine MA, Bhide SV. Chemopreventive effect of turmeric against stomach and skin tumors induced by chemical carcinogens in Swiss mice. Nutr Cancer. 1992;17(1):77-83.

9. Dorai T, Cao YC, Dorai B, Buttyan R, Katz AE. Therapeutic potential of curcumin in human prostate cancer. III. Curcumin inhibits proliferation, induces apoptosis, and inhibits angiogenesis of LNCaP prostate cancer cells in vivo. Prostate. 2001;47(4):293-303.

10. Zhang J, Tang Q, Xu X, Li N. Development and evaluation of a novel phytosome-loaded chitosan microsphere system for curcumin delivery. Int J Pharm. 2013;448(1):168-174.

11. Jang DJ, Kim ST, Oh E, Lee K. Enhanced oral bioavailability and antiasthmatic efficacy of curcumin using redispersible dry emulsion. Biomed Mater Eng. 2014;24(1):917-930.

12. Seo SW, Han HK, Chun MK, Choi HK. Preparation and pharmacokinetic evaluation of curcumin solid dispersion using Solutol ${ }^{\circledR}$ HS15 as a carrier. Int J Pharm. 2012;424(1-2):18-25.

13. Li L, Braiteh FS, Kurzrock R. Liposome-encapsulated curcumin: in vitro and in vivo effects on proliferation, apoptosis, signaling, and angiogenesis. Cancer. 2005;104(6):1322-1331.

14. Michel D, Chitanda JM, Balogh R, et al. Design and evaluation of cyclodextrin-based delivery systems to incorporate poorly soluble curcumin analogs for the treatment of melanoma. Eur J Pharm Biopharm. 2012;81(3):548-556.

15. Singh R, Kristensen S, Tønnesen HH. Influence of cosolvents, ionic strength and the method of sample preparation on the solubilization of curcumin by Pluronics and HP-gamma-cyclodextrin. Studies of curcumin and curcuminoids, XLIV. Pharmazie. 2012;67(2):131-142.

16. Gangwar RK, Tomar GB, Dhumale VA, Zinjarde S, Sharma RB, Datar S. Curcumin conjugated silica nanoparticles for improving bioavailability and its anticancer applications. J Agric Food Chem. 2013;61(40):9632-9637.

17. Dilnawaz F, Sahoo SK. Enhanced accumulation of curcumin and temozolomide loaded magnetic nanoparticles executes profound cytotoxic effect in glioblastoma spheroid model. Eur J Pharm Biopharm. 2013;85(3 Pt A):452-462.

18. Esposito E, Ravani L, Mariani P, et al. Effect of nanostructured lipid vehicles on percutaneous absorption of curcumin. Eur J Pharm Biopharm. 2014;86(2):121-132.

19. Thapa RK, Baskaran R, Madheswaran T, Kim JO, Yong CS, Yoo BK. In vitro release and skin permeation of tacrolimus from monoolein-based liquid crystalline nanoparticles. J Drug Deliv Sci Technol. 2012;22(6): $457-576$.

20. Thapa RK, Baskaran R, Madheswaran T, Kim JO, Yong CS, Yoo BK. Preparation, characterization, and release study of tacrolimus-loaded liquid crystalline nanoparticles. J Dispers Sci Technol. 2013;34(1):72-77.

21. Madheswaran T, Baskaran R, Thapa RK, et al. Design and in vitro evaluation of finasteride-loaded liquid crystalline nanoparticles for topical delivery. AAPS Pharm Sci Tech. 2013;14(1):45-52.

22. Dong YD, Larson I, Hanley T, Boyd BJ. Bulk and dispersed aqueous phase behavior of phytantriol: effect of vitamin $\mathrm{E}$ acetate and F127 polymer on liquid crystal nanostructure. Langmuir. 2006;22(23): 9512-9518.

23. Anand P, Kunnumakkara AB, Newman RA, Aggarwal BB. Bioavailability of curcumin: problems and promises. Mol Pharm. 2007;4(6): $807-818$.

24. Cheng AL, Hsu CH, Lin JK, et al. Phase I clinical trial of curcumin, a chemopreventive agent, in patients with high-risk or pre-malignant lesions. Anticancer Res. 2001;21(4B):2895-2900.

25. Sharma RA, McLelland HR, Hill KA, et al. Pharmacodynamic and pharmacokinetic study of oral Curcuma extract in patients with colorectal cancer. Clin Cancer Res. 2001;7(7):1894-1900.

26. Shoba G, Joy D, Joseph T, Majeed M, Rajendran R, Srinivas PS. Influence of piperine on the pharmacokinetics of curcumin in animals and human volunteers. Planta Med. 1998;64(4):353-356.

27. Ireson C, Orr S, Jones DJ, et al. Characterization of metabolites of the chemopreventive agent curcumin in human and rat hepatocytes and in the rat in vivo, and evaluation of their ability to inhibit phorbol ester-induced prostaglandin E2 production. Cancer Res. 2001;61(3):1058-1064. 
28. Ireson CR, Jones DJ, Orr S, et al. Metabolism of the cancer chemopreventive agent curcumin in human and rat intestine. Cancer Epidemiol Biomarkers Prev. 2002;11(1):105-111.

29. Ravindranath V, Chandrasekhara N. Absorption and tissue distribution of curcumin in rats. Toxicology. 1980;16(3):259-265.

30. Wang YJ, Pan MH, Cheng AL, et al. Stability of curcumin in buffer solutions and characterization of its degradation products. J Pharm Biomed Anal. 1997;15(12):1867-1876.

31. Tønnesen HH, Másson M, Loftsson T. Studies of curcumin and curcuminoids. XXVII. Cyclodextrin complexation: solubility, chemical and photochemical stability. Int J Pharm. 2002;244(1-2):127-135.

32. Madheswaran T, Baskaran R, Yong CS, Yoo BK. Enhanced topical delivery of finasteride using glyceryl monooleate-based liquid crystalline nanoparticles stabilized by cremophor surfactants. AAPS Pharm Sci Tech. 2014;15(1):44-51.

33. Murgia S, Bonacchi S, Falchi AM, et al. Drug-loaded fluorescent cubosomes: versatile nanoparticles for potential theranostic applications. Langmuir. 2013;29(22):6673-6679.
34. Yadav VR, Prasad S, Kannappan R, et al. Cyclodextrin-complexed curcumin exhibits anti-inflammatory and antiproliferative activities superior to those of curcumin through higher cellular uptake. Biochem Pharmacol. 2010;80(7):1021-1032.

35. Atsumi T, Fujisawa S, Tonosaki K. Relationship between intracellular ROS production and membrane mobility in curcumin- and tetrahydrocurcumin-treated human gingival fibroblasts and human submandibular gland carcinoma cells. Oral Dis. 2005;11(4):236-242.

36. Ingolfsson HI, Koeppe RE, Andersen OS. Curcumin is a modulator of bilayer material properties. Biochemistry. 2007;46(36):10384-10391.

37. Chen H, Zhang ZS, Zhang YL, Zhou DY. Curcumin inhibits cell proliferation by interfering with the cell cycle and inducing apoptosis in colon carcinoma cells. Anticancer Res. 1999;19(5A):3675-3680.

38. Hanif R, Qiao L, Shiff SJ, Rigas B. Curcumin, a natural plant phenolic food additive, inhibits cell proliferation and induces cell cycle changes in colon adenocarcinoma cell lines by a prostaglandin-independent pathway. J Lab Clin Med. 1997;130(6):576-584.
International Journal of Nanomedicine

\section{Publish your work in this journal}

The International Journal of Nanomedicine is an international, peerreviewed journal focusing on the application of nanotechnology in diagnostics, therapeutics, and drug delivery systems throughout the biomedical field. This journal is indexed on PubMed Central, MedLine, CAS, SciSearch $\AA$, Current Contents ${ }^{\circledR} /$ Clinical Medicine,

\section{Dovepress}

Journal Citation Reports/Science Edition, EMBase, Scopus and the Elsevier Bibliographic databases. The manuscript management system is completely online and includes a very quick and fair peer-review system, which is all easy to use. Visit http://www.dovepress.com/ testimonials.php to read real quotes from published authors. 